\title{
New Mechanism and Analytical Formula for Understanding the Gravity Constant $G$
}

\author{
Nader Butto \\ Rabin Medical Centre, Petah-Tikva, Israel \\ Email: nader.butto@gmail.com
}

How to cite this paper: Butto, N. (2020) New Mechanism and Analytical Formula for Understanding the Gravity Constant $G$. Journal of High Energy Physics, Gravitation and Cosmology, 6, 357-367. https://doi.org/10.4236/jhepgc.2020.63029

Received: April 10, 2020

Accepted: July 5, 2020

Published: July 8, 2020

Copyright $\odot 2020$ by author(s) and Scientific Research Publishing Inc. This work is licensed under the Creative Commons Attribution International License (CC BY 4.0).

http://creativecommons.org/licenses/by/4.0/

(c) (i) Open Access

\begin{abstract}
The nature of gravitation and $G$ is not well understood. A new gravitation mechanism is proposed that explains the origin and essence of the gravitational constant, $G$. Based on general relativity, the vacuum is considered to be a superfluid with measurable density. Rotating bodies drag vacuum and create a vortex with gradient pressure. The drag force of vacuum fluid flow in the arm of the vortex is calculated relative to the static vacuum and a value that is numerically equal to that of $G$ is obtained. Using Archimedes' principle, it is determined that $G$ is the volume of vacuum displaced by a force equivalent to its weight which is equal to the drag force of the vacuum. It is concluded that the gravitational constant $G$ expresses the force needed to displace a cubic metre of vacuum that weighs one $\mathrm{kg}$ in one second. Therefore, $G$ is not a fundamental physical constant but rather is an expression of the resistance encountered by the gravitational force in the vacuum.
\end{abstract}

\section{Keywords}

Gravitational Constant, Vacuum Density, Drag Force, Vortex Formation, Specific Volume Flow, Archimedes' Principle

\section{Introduction}

The gravitational constant denoted by the letter $G$, is an empirical physical constant pivotal in the calculation of gravitational effects in Newton's law of universal gravitation and in Albert Einstein's general theory of relativity.

Gravity is most accurately described by the general theory of relativity (proposed by Albert Einstein in 1915), which describes gravity not as a force but as a consequence of the curvature of space-time caused by the uneven distribution of mass. The most extreme example of this curvature of space-time is a black hole, from which nothing can escape once past its event horizon, not even light [1]. 
In 1687, Newton published Principia, which hypothesises the inverse-square law of universal gravitation [2].

Newton's law states that every object in the universe attracts every other object with a force which, for any two bodies, is proportional to their mass and varies inversely as the square of the distance between them. This statement is expressed mathematically by the following well-known equation:

$$
F_{g}=G\left(m_{1} \cdot m_{2} / r^{2}\right)
$$

where $m_{1}$ and $m_{2}$ are the interacting masses and $r$ is their relative distance vector. The Newtonian constant of gravitation $G$, is typically assumed to be a universal constant whose measured value is $6.67408(31) \times 10^{-11} \mathrm{~m}^{3} \cdot \mathrm{kg}^{-1} \cdot \mathrm{s}^{-2} \quad$ [3]

The numerical value of $G$ was initially determined by English physicist Cavendish in 1798 through the measurement of the attractive force between two spheres with the aid of a torsion balance.

More than three hundred and fifty years after the discovery of gravity by Newton, there is still no theoretical explanation for the mechanism of gravity. As a result, the true nature of gravity and the essence of $G$ are not understood. It is unknown whether the origin of $G$ can be described using an analytical formula [4].

The current spread of values is approaching $0.05 \%$, which is more than 10 times the uncertainties on each measurement, and it therefore appears that we know $G$ only to three significant figures! This is very poor compared with other physical constants, many of which have uncertainties of the order of parts in $10^{8}$ [5].

Determining the ultimate physical origin of gravity and its associated constant $G$ could provide important insights into a fundamental understanding of the universe.

In this article, the origin and essence of $G$ is described and a mathematical formula is derived to calculate its value.

The starting point is the superfluid vacuum that has calculable density based on universe expanding measurements and Hubble constant determination. Then gravity is described as the result of the dragging force of the rotating planet that generates a vortex that curves space and time and generates pressure gradient and vacuum flow to the centre of the vortex. Calculation of the dragging force of the flow with the vacuum gives us the same value of constant $G$.

\section{The superfluid nature of the vacuum}

Models that describe the theory of gravity based on the idea that the physical space could be "filled" with a constitutive continuum medium characterised by specific properties have been previously proposed. The vacuum could therefore be comprised of a fundamental substrate (on the quantum scale) such as an elastic solid-state medium, a fluid, or a Higgs condensate [6] [7] [8] [9].

According to the superfluid theory of vacuum, the physical vacuum is described as a quantum superfluid, which behaves like a fluid with minimal viscosity and with extremely high thermal conductivity. It is a perfect fluid in the sense that it is non-particulate and has no structural memory. If perturbed, it has no tendency to revert to its former physical state. 
The superfluid vacuum theory proposes a mass generation mechanism that may replace or supplement the electroweak Higgs mechanism. It has been shown that the masses of elementary particles could arise because of their interaction with a superfluid vacuum. This phenomenon is similar to the gap generation mechanism in superconductors [10].

Furthermore, the first postulate of general relativity states that the source of a gravitational field is the stress-energy tensor of a perfect fluid [11]. This 'stress-energy tensor' contains four non-zero components, i.e., the density of the perfect fluid and the pressure of the perfect fluid in each of the three physical axes. According to general relativity, a perfect fluid is defined as a fluid with no viscosity or heat conduction.

\section{Density of the Vacuum}

Although there is no consensus regarding vacuum density, its value primarily depends on general relativity. It is therefore possible to measure the energy density of the vacuum through astronomical observations that determine the curvature of space-time and the expansion of the universe.

The measurement of universal expansion based on the relation between galaxy velocity $(v)$ and its distance $(d)[12]$

$$
v=H o \times d .
$$

This relation is the well-known Hubble Law. It indicates a constant expansion of the cosmos, where galaxies recede from each other at a constant speed per unit distance; thus, more distant objects move faster than nearby ones.

The expansion of the universe has been studied by several different methods. The Wilkinson Microwave Anisotropy Probe (WMAP) mission completed in 2003 , represents a major step toward precision in determining the expansion of the universe and calculating vacuum density [13].

Another method is using the Baryon Oscillation Spectroscopic Survey (BOSS) [14] by studying more than 140,000 extremely bright galaxies known as quasars, which serve as a "standard ruler", scientists map density variations in the universe. By nearly tripling the number of quasars previously studied, as well as implementing a new technique, the scientists were able to calculate the expansion rate to 42 miles (68 kilometres) per second per 1 million light-years with greater precision, while looking farther back in time.

It is important to note that the study of the expansion rate of the universe has shown that the universe is close to critical density. Critical density is the value at which the Universe is balanced and expansion is halted.

The density is typically expressed as a fraction of the density required for the critical condition to be fulfilled through the use of a parameter known as omega $(\Omega)$ where $\Omega=\rho / \rho_{\text {critical }}$. The limiting critical density is described by $\Omega=1$. For a value of omega less than 1 (known as an "open universe"), the final fate of the universe is a "cold death". In this case, the universe expands forever, albeit at an ever-decreasing rate. For omega greater than 1, the universe is "closed" and 
will at some point collapse in on itself and end in a "big crunch". For omega equal to 1 , the universe is called "flat"; this universe has a critical density and expansion is halted only after an infinite time. Currently, the estimated sum of the contributions to the total density parameter, $\Omega_{0}$, is $\Omega_{0}=1.02 \pm 0.02$, which indicates that the universe is close to critical density.

Hence, the critical density that defines the boundary condition between the open and closed solutions of the standard cosmological model is [15]

$$
\rho_{c r}=3 H_{0}^{2} / 8 \pi G=1.88\left(h^{2}\right) \times 10^{-29} \mathrm{~g} / \mathrm{cm}^{3},
$$

where $\rho_{c r}$ is the critical density, $H$ is the current value of the Hubble constant and $h \equiv H_{0} / 71 \mathrm{~km} \cdot \mathrm{sec}^{-1} \cdot \mathrm{Mpc}^{-1} \cdot H_{0}=71 \mathrm{~km} \cdot \mathrm{s}^{-1} \cdot \mathrm{Mpc}^{-1} \quad$ (WMAP value for the Hubble parameter [16]).

The infrared camera was installed on the Hubble Telescope in 2009, and the astronomical measurements used to calculate the Hubble constant obtained a slightly higher value with narrow error bars.

The Hubble Space Telescope can determine the distances to Milky Way Cepheids (a type of variable star) through accurately separating their spectra from the bluer stars that tend to surround cepheids. In a recent publication, Riess [17] reported that

$$
H_{0}=73.24 \mathrm{~km} \cdot \mathrm{s}^{-1} \cdot \mathrm{Mpc}^{-1},
$$

where $\mathrm{Mpc}$ is equal to $3.09 \times 10^{19} \mathrm{~km}$.

The most recent result published in this year (2017) [18], and the cosmological density $\rho_{c}$ (with small uncertainty) is therefore calculated to be

$$
\rho_{c, 0}=3 H_{0}^{2} / 8 \pi G=11.11( \pm 1.05) \times 10^{-27} \mathrm{~kg} / \mathrm{m}^{3} .
$$

\section{Mechanism of Gravitation}

According to general relativity, the gravitational attraction observed between masses results from the warping of space and time by these masses. The gravitational potential generated by a mass, which depends on the radial distance from the mass, affects the running rate of clocks, the measurement of distances and the velocity of light. This fact is theoretically explained within general relativity and supported by strong experimental evidence. Nevertheless, there is no description of causation as a curvature of space.

In this work, a new model that explains the gravitational force and the curvature of space-time is proposed. The interaction of rotating masses within the vacuum leads to a drag effect, vortex formation, space-time curvature and resultant gravitational force. All particles and celestial bodies are immersed in this fluid vacuum. All particles of matter spin and exert a drag effect on the surrounding vacuum. This creates a small vortex around the particle even if it is located within a stationary body. The sum of the effects due to all the constituent particles creates a gravitational force arising from the mass. In a rotating celestial body, the rotating particles have translational speed that drags the surrounding vacuum. It follows from 
the case of the "normal" drag force for an object moving in a linear fashion, such that rotational motion creates a vortex around the celestial body.

The vortex resulting from the rotating mass creates a dynamic pressure that is lowest in the core region closest to the vortex core. This pressure increases with distance from the centre of the vortex. This behaviour is in accordance with Bernoulli's principle, which states that for an inviscid flow, an increase in the speed of the fluid occurs simultaneously with a decrease in pressure or a decrease in the fluid's potential energy. The gradient of this pressure forces the fluid to curve around the central axis of the vortex. This dynamic pressure causes the gravitational force and is proportional to the square of the distance "r," from the axis according to the inverse-square law. This law states that a specified physical quantity or intensity is inversely proportional to the square of the distance from the source of that physical quantity.

The flow in the arms of the vortex (created by the gradient pressure) travels at the speed of light relative to the static surrounding vacuum. This creates a drag between static vacuum and vortex arm flow.

Drag is a common term which refers to any force that opposes motion. When a fluid moves in a fluid such as a vortex, it experiences two forms of drag force. Forces normal to the motion are referred to as pressure drag and shear forces due to flow along surfaces "parallel" to the motion (edges) are referred to as viscous drag.

Pressure drag is caused by molecules hitting a surface and returning. This causes a change in linear momentum and results in normal force. Viscous drag results from the attraction between molecules due to the relative velocity between flux and static fluid.

The drag force on any object is proportional to the density of the fluid and the square of the relative flow speed between the moving object and static fluid, according to the following formula:

$$
F_{d}=\frac{1}{2} \rho v^{2} A C_{D}
$$

where $F_{d}$ is the drag force (which is defined as the force component in the direction of flow velocity [19]), $\rho$ is the mass density of the fluid, $v$ is the flow velocity relative to the object, $A$ is the interaction area and $C_{D}$ is the drag coefficient.

In fluid dynamics, the drag coefficient is a dimensionless quantity that is used to quantify the viscous drag or shear forces on an object in a fluid environment. The drag coefficient is the ratio of drag force to the product of area and the force generated by dynamic pressure.

$$
C_{D}=F_{d} / \frac{1}{2} \rho v^{2} A .
$$

However, if there are no data on the drag coefficient and drag force, the above mentioned formula cannot be used to determine the drag coefficient.

Another method of calculating the drag coefficient is by using the Reynolds number. The drag coefficient of an object is regarded as a function of the Rey- 
nolds number, based on the relative velocity between the rotating object and surrounding fluid. Reynolds number is the ratio of inertial (resistance to change or motion) forces to viscous forces [20].

$$
\begin{gathered}
\operatorname{Re}=\left(\rho u^{2}\right) /(\mu u / L), \\
\operatorname{Re}=\rho u L / \mu, \\
\operatorname{Re}=u L / v,
\end{gathered}
$$

where $R e=$ Reynolds Number (non-dimensional), $\rho=\operatorname{density~}\left(\mathrm{kg} / \mathrm{m}^{3}\right), u=$ is the mean velocity of flow relative to vacuum (speed of light; SI units: $\mathrm{m} / \mathrm{s}$ ), $\mu=\mathrm{dy}$ namic viscosity $\left(\mathrm{Ns} / \mathrm{m}^{2}\right), L=$ characteristic length $(\mathrm{m})$ and $v=\mu / \rho=$ kinematic viscosity $\left(\mathrm{m}^{2} / \mathrm{s}\right)$.

In general, the drag coefficient is not an absolute constant for a given body shape. Larger velocities, larger objects and lower viscosities (such as that in this case) contribute to larger Reynolds numbers [21].

While there is no theorem that relates the Reynolds number to turbulence, flows at Reynolds numbers larger than 5000 are typically turbulent, while those at low Reynolds numbers are laminar.

As the Reynolds number increases, inertial forces become stronger than viscous forces, and a laminar boundary layer is generated. Therefore, the drag coefficient decreases as the Reynolds number increases.

The graph of $C_{D}$ vs. $R e$ is shown in Figure 1.

According to Figure 1, the value of the drag coefficient can be estimated. Planets and large celestial bodies have large Reynolds numbers. It can therefore be deduced that these objects have drag coefficients that lie between 0.1 and 0.2 .

\section{Drag Force of the Vacuum}

If the vacuum is considered as a liquid that travels at the speed of light and given its density, the drag force can be calculated by applying the drag force formula.

The drag force equation is transformed to the pressure equation by dividing both sides by area to obtain:

$$
F_{d} / A=P=\frac{1}{2} \rho v^{2} C_{D}
$$

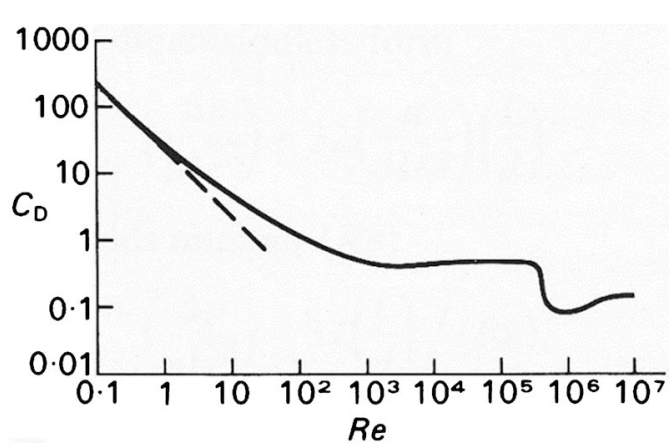

Figure 1. $C_{D}$ vs. $R e$ for a sphere. The dashed curve represents theoretical results for small values of $\operatorname{Re}$ [22]. 
where $P$ is the pressure gradient generated by drag. The drag pressure of the vacuum is constant because it is derived through conservation of momentum using density and velocity.

If the vacuum density is $11.11 \times 10^{-27} \mathrm{~kg} / \mathrm{m}^{3}, \quad v=c=3 \times 10^{8} \mathrm{~m} / \mathrm{s}$ and the drag coefficient is between 0.1 and 0.2 .

Substituting for $\rho, v^{2}$ and $C_{D}(0.13349)$ in Equation (9) gives:

$$
P=6.67383255 \times 10^{-11} \mathrm{~kg} / \mathrm{m} \cdot \mathrm{s}^{2} \text { or } \mathrm{N} / \mathrm{m}^{2}
$$

The resulting answer has the same numerical value as G, i.e., $(6.67384 \pm$ $0.00080) \times 10^{-11} \mathrm{~m}^{3} / \mathrm{kg} \cdot \mathrm{s}$ or $\mathrm{N} \cdot \mathrm{m}^{2} / \mathrm{kg}^{2}$.

This drag pressure is the pressure required to move the equivalent specific volume per second (specific volume rate). In fluid mechanics, a fluid is displaced when an object is immersed in it i.e., the object displaces the fluid and occupies its space. In this case, drag force generated by gradient pressure is what displaces the vacuum fluid. The volume of vacuum that is displaced is equivalent to the volume of fluid flow generated by gradient pressure.

In thermodynamics, specific volume is defined as the number of cubic metres occupied by one kilogram of a particular substance or the ratio of a substance's volume to its mass. It is the reciprocal of density and it is an intrinsic property of matter. The standard unit of specific volume is the cubic metre per kilogram $\left(\mathrm{m}^{3} \cdot \mathrm{kg}^{-1}\right)$. Specific volume rate is defined as the number of cubic metres occupied by one kilogram of a particular substance that flows, per unit time. This is equivalent to the flow rate per unit weight because $\mathrm{m}^{3} / \mathrm{s}$ represents flow rate.

According to Archimedes' principle, the weight of a displaced fluid is directly proportional to its volume. The magnitude of force required to counteract flow is equal to the weight of the displaced fluid. Therefore, the rate of displacement of specific volume per kg per second ( $G$ constant) would be proportional to the pressure needed to move the weight of that volume, which is equivalent to the drag force.

Therefore, $G$ is the resistance force that gravity must overcome in order to move the weight of one cubic metre of vacuum.

\section{Discussion}

In this work, a new mechanism of gravity related to the rotation of a planet in a superfluid vacuum has been proposed. Rotating planets drag vacuum energy around their particles and curve space-time by creating vortex flow. Although this idea is not new because general relativity predicts that the rotation of planets drags superfluid vacuum and 'warps' space-time, there is no explanation for the curvature of space-time. In the proposed model, curved space-time is the effect and not the cause of gravity.

According to the proposed model, a rotating planet drags vacuum from all directions toward its centre and creates a vortex with a pressure gradient that attracts the vacuum fluid from the periphery to the centre of the vortex. Therefore, the vacuum superfluid flow from the periphery to the centre of the vortex 
"curves" space-time and generates the pressure that pushes the objects forward to the centre of the vortex. Objects obstructing the flow will be pushed to the centre of the vortex. This is the origin of the gravitational force.

The vortex model is not new. A large number of philosophers used the idea of cosmic vortices in their explanation of creation. In the ancient world, these philosophers included Empedocles, Leucippus, Democritus and Aristotle. During the Renaissance, this idea was developed by R. Descartes, J. MacCullagh, J. J. Thomson and W. Thomson (Lord Kelvin). However, these scientists could not formulate their ideas in a rigorous and mathematical form and instead their findings were formulated as philosophical speculations. In recent years, different theories proposed the existence of ether vortex mechanisms as the cause of gravity, such as the vortex gravitation model [23]. However, there is no explanation of the mechanism of vortex formation. Furthermore, the theory does not predict, calculate, or describe the essence of $G$.

In this model, the drag force created by the interaction between a mass and the surrounding vacuum is considered to be the origin of vortex formation.

The drag theory of gravity was originally proposed by Nicolas Fatio de Duillier in 1690 and later by Georges-Louis Le Sage in 1748 [24]. They proposed a mechanical explanation for Newton's gravitational force in terms of streams of minute unobservable particles impacting all material objects from all directions.

According to this model, any two material bodies partially shield each other from impinging corpuscles. This results in a net imbalance in the pressure exerted by the impact of the corpuscles on the bodies, which tends to drive the bodies together. This mechanical explanation for gravity was not widely accepted, although it continued to be studied occasionally by physicists until the beginning of the $20^{\text {th }}$ century. However, by this time it was considered to be definitively discredited [25].

According to general relativity, planet rotation is dragged by an unknown force. Such a "drag" implies that there is friction in the motion of space-time with respect to a mass where "inertial dragging" occurs. General relativistic formulations show the requirement of tangential motion when the continuum is assumed to be a superfluid. The explanation of inertial dragging does not provide an identifying cause based on a fundamental theory [26]. The inertial dragging is explained by the theory of vortex space rotation. This states that the gravitational force is independent of the masses and densities of bodies. The masses of the planets have therefore been determined based on the law of angular momentum conservation as they were created at the centres of space torsions through matter accumulation. The same force that created celestial bodies continues to exist and it exerts inertial dragging that maintains their rotation.

This theory does not exclude a drag force as the causes the rotation of celestial bodies. However, vortex formation caused by a rotating mass is proposed. In both scenarios, there is vortex formation and associated pressure gradient that attracts vacuum fluid to the centre of the planet as demonstrated by experiments 
of rotating spheres in liquid.

In order to obtain $G$, the drag force of the flowing vacuum fluid is calculated relative to the static surrounding vacuum that depends primarily on the density of the vacuum and the square of velocity of the flow according to dragging force formula.

\section{Conclusions}

The results presented here strongly suggest that $G$ is related to the structure and properties of the physical vacuum, where the vacuum is considered as a medium characterised by specific properties such as density, viscosity and speed. The density of vacuum is calculated based on the current value of the Hubble constant.

A new mechanism of gravity is proposed according to which gravitational force is the result of the gradient pressure of the vacuum that is generated by the drag force of a rotating planet. The drag force of a vortex created by a rotating body is calculated, and this is found to have the same numerical value as $G$.

It is therefore concluded that the gravitational force is a "push force" that delivers a part of its momentum to a mass upon colliding with it and pushes it forward toward the vortex centre. However, the gravitational force is diminished by the $G$ value that represents the resistance of the vacuum to the gradient flow of the vortex. Therefore, $G$ is not a fundamental physical constant instead it is an expression of the resistance encountered by the gravitational force in the vacuum.

This paper proposes a new approach to understand gravity. Hence, it will significantly contribute to understand the mechanism of gravitation.

\section{Limitation}

The density value used to calculate the drag force is based on recent astronomical measurements. This value, which has been determined as $11.11 \times 10^{-27} \mathrm{~kg} / \mathrm{m}^{3}$ still has an associated uncertainty of $( \pm 1.05)$, which can result in a significant change in the value of G. Furthermore, although the drag coefficient value used to calculate the drag force was within the range values, it is an estimated value. Further research is therefore needed to confirm this theory.

\section{Acknowledgements}

The author would like to thank Enago (https://www.enago.com/) for the English language review.

This research did not receive any specific grant from funding agencies in the public, commercial, or not-for-profit sectors.

\section{Conflicts of Interest}

The author declares no conflicts of interest regarding the publication of this paper.

\section{References}

[1] Poynting, J.H. (1911) Gravitationin Chisholm, Hugh. Encyclopædia Britannica. Vol. 
12, 11 th Edition.

[2] Podlaha, M.F. and Sjodin, T. (1984) On Universal Fields and de Broglie's Waves. Nuovo Cimento B, 79, 85. https://doi.org/10.1007/BF02723840

[3] The NIST Reference on Constants, Units and Uncertainty. https://physics.nist.gov/cgi-bin/cuu/Value?bg

[4] Winterberg, F. (1988) Substratum Approach to a Unified Theory of Elementary Particles. Zeitschrift für Naturforschung A, 43, 1131-1150. https://doi.org/10.1515/zna-1988-1219

[5] Quinn, T. and Speake, C. (2014) The Newtonian Constant of Gravitation-A Constant Too Difficult to Measure? An Introduction. Philosophical Transactions of the Royal Society A Mathematical Physical and Engineering Sciences, 372, Article ID: 20140253. https://doi.org/10.1098/rsta.2014.0253

[6] Dmitriev, V.P. (1992) The Elastic Model of Physical Vacuum. Mechanics of Solids, 26, 60-71.

[7] Consoli, M.A. (2002) Weak, Attractive, Long Range Force in Higgs Condensates. Physics Letters B, 541, 307-313. https://doi.org/10.1016/S0370-2693(02)02236-0

[8] Liberati, S. and Maccione, L. (2014) Astrophysical Constraints on Planck Scale Dissipative Phenomena. Physical Review Letters, 112, Article ID: 151301. https://doi.org/10.1103/PhysRevLett.112.151301

[9] Zloshchastiev, K.G. (2011) Spontaneous Symmetry Breaking and Mass Generation as Built-In Phenomena in Logarithmic Nonlinear Quantum Theory. Acta Physica Polonica B, 42, 261-292.

[10] Avdeenkov, A.V. and Zloshchastiev, K.G. (2011) Quantum Bose Liquids with Logarithmic Nonlinearity: Self-Sustainability and Emergence of Spatial Extent. Journal of Physics B: Atomic, Molecular and Optical Physics, 44, Article ID: 195303. https://doi.org/10.1088/0953-4075/44/19/195303

[11] Schutz, B.F. (2009) A First Course in General Relativity. Sections 4.6 \& 4.7, Cambridge University Press, Cambridge. https://doi.org/10.1017/CBO9780511984181

[12] Hubble, E.A. (1929) Relation between Distance and Radial Velocity among Extra-Galactic Nebulae. Proceedings of the National Academy of Sciences of the United States of America, 15, 168-173. https://doi.org/10.1073/pnas.15.3.168

[13] Wilkinson Microwave Anisotropy Probe. http://map.gsfc.nasa.gov

[14] Dawson, K.S., et al. (2013) The Baryon Oscillation Spectroscopic Survey (BOSS) of SDSS-III. Astronomical Journal, 145, 10.

[15] Oldershaw, R.L. (1987) The Self-Similar Cosmological Paradigm-A New Test and Two New Predictions. Astronomical Journal, 322, 34-36. https://doi.org/10.1086/165699

[16] Tegmark, M., et al. (2004) Cosmological Parameters from SDSS and WMAP. Physical Review D, 69, Article ID: 103501.

[17] Riess, A.G., et al. (2016) A 2.4\% Determination of the Local Value of the Hubble Constant. The Astrophysical Journal, 826, 56. https://doi.org/10.3847/0004-637X/826/1/56

[18] Bonvin, V., et al. (2017) HOLiCOW-V. New COSMOGRAIL Time Delays of HE 0435-1223: H0 to 3.8 Percent Precision from Strong Lensing in a Flat $1 \mathrm{CDM}$ Model. Monthly Notices of the Royal Astronomical Society, 465, 4914-4930. https://doi.org/10.1093/mnras/stw3006

[19] Drag Equation. https://en.wikipedia.org/wiki/Drag_equation\#cite_note-1 
[20] Reynolds Number.

https://en.wikipedia.org/wiki/Reynolds_number\#cite_note-NASA-4\#cite_note-NAS A-4

[21] Fox \& McDonald (1973) Introduction to Fluid Mechanics. John Wiley, New York, 406.

[22] Clancy, L.J. (1975) Aerodynamics, Section 4.17. John Wiley \& Sons, Hoboken.

[23] Orlov, S. (2012) Foundation of Vortex Gravitation, Cosmology and Cosmogony. International Journal of Astronomy, 1, 1-16.

[24] Le Sage's Theory of Gravitation. https://en.wikipedia.org/wiki/Le_Sage\%27s_theory_of_gravitation

[25] Edwards, M.R. (2002) Pushing Gravity: New Perspectives on Le Sage's Theory of Gravitation (Illustrated ed.). Indiana University, Bloomington, 65.

[26] Islam, J.N. (1985) Rotating Fields in General Relativity. 6th Edition, Cambridge University Press, Cambridge, 18-20. https://doi.org/10.1017/CBO9780511735738 